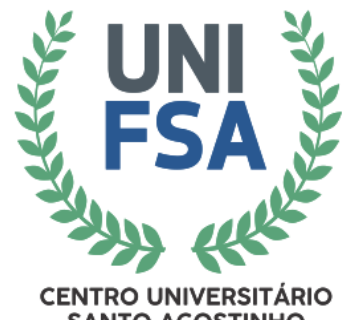

SANTO ACOSTINHO
CENTRO UNIVERSITÁRIO SANTO ACOSTINHO

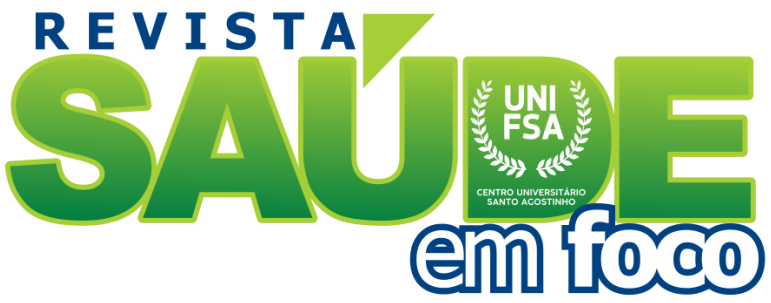

www4.fsanet.com.br/revista

Revista Saúde em Foco, Teresina, v. 8, n. 3, art. 4, p. 54-67, set./dez. 2021

ISSN Eletrônico: 2358-7946

http://dx.doi.org/10.12819/rsf.2021.8.3.4

\title{
A Importância da Manutenção da Saúde Bucal em Pacientes Hospitalizados
}

The Importance of Oral Health Maintenance in Hospitalized Patients

Maria Olinda Vieira Cavalcante Acadêmica de Odontologia pelo Centro Universitário Facid|Wyden

E-mail: mariolacavalcante@ hotmail.com

Marcia Socorro da Costa Borba

Doutora em Clínica Odontológica pela Universidade Estadual de Campinas Professora do Centro Universitário Facid|Wyden

E-mail: marcia.borba@ facid.edu.br

Marina Barbosa Ferreira Santos

Acadêmica de Odontologia pelo Centro Universitário Facid|Wyden

E-mail: marina.10@ hotmail.com

Agnes Lorena Araújo da Gama

Acadêmica de Odontologia pela Universidade Estadual do Piauí

E-mail: lorenagama_8@hotmail.com

Lucas Ludugério Lunas

Acadêmico de Odontologia pelo Centro Universitário Facid|Wyden

E-mail: lunaslucas0@ hotmail.com

Endereço: Maria Olinda Vieira Cavalcante

Centro Universitário UniFacid/Wyden, Rua Veterinário

Bugyja Brito, $n^{\circ}$ 1354, Bairro Horto Florestal Teresina -

PI Brasil.

Endereço: Marcia Socorro da Costa Borba

Centro Universitário UniFacid/Wyden, Rua Veterinário Bugyja Brito, $n^{\circ} 1354$, Bairro Horto Florestal Teresina PI Brasil.

Endereço: Marina Barbosa Ferreira Santos

Centro Universitário UniFacid/Wyden, Rua Veterinário Bugyja Brito, $n^{\circ} 1354$, Bairro Horto Florestal Teresina PI Brasil.

Endereço: Agnes Lorena Araújo da Gama

Centro Universitário UniFacid/Wyden, Rua Veterinário Bugyja Brito, $\mathrm{n}^{\circ}$ 1354, Bairro Horto Florestal Teresina PI Brasil.

Endereço: Lucas Ludugério Lunas

Centro Universitário UniFacid/Wyden, Rua Veterinário Bugyja Brito, ${ }^{\circ} 1354$, Bairro Horto Florestal Teresina PI Brasil.
Editor-Chefe: Dr. Tonny Kerley de Alencar Rodrigues

Artigo recebido em 16/10/2021. Última versão recebida em 27/10/2021. Aprovado em 28/10/2021.

Avaliado pelo sistema Triple Review: a) Desk Review pelo Editor-Chefe; e b) Double Blind Review (avaliação cega por dois avaliadores da área).

Revisão: Gramatical, Normativa e de Formatação 


\title{
RESUMO
}

É válido ressaltar que na maioria dos centros de saúde muitas questões sobre higiene bucal são negligenciadas. Além disso, estudos recentes demonstraram que o cuidado com a saúde bucal se mostrou eficaz no controle de patógenos dos pacientes e os protocolos de cuidados sistematizados otimizaram a prestação do serviço. A área da Odontologia Hospitalar, como um todo, tem a capacidade de diminuir o tempo de internação de pacientes, riscos de diversas infecções e, sem dúvidas, proporcionar a qualidade de vida da pessoa doente. O estudo objetivou buscar, na literatura, embasamentos que fundamentassem a discussão sobre a importância do cuidado acerca da saúde bucal em pacientes hospitalizados. As bases de dados empregadas para o rastreamento dos artigos foram MEDLINE, BBO - Odontologia / LILACS e Scielo, utilizando-se na busca a combinação das palavras "Saúde bucal"; "Pacientes Internados"; "Unidades de terapia intensiva" e " Odontologia Hospitalar". As patologias bucais são importantes fontes de agravamento sistêmico do paciente e devem ser prevenidas e solucionadas por um cirurgião dentista capacitado a trabalhar em ambiente hospitalar. Os protocolos de conduta são eficientes para agilizarem e darem qualidade à assistência da saúde do paciente hospitalizado.

Palavras-chaves: Saúde Bucal. Pacientes Internados. Unidades de Terapia Intensiva. Odontologia Hospitalar.

\begin{abstract}
It is worth noting that in most health centers many questions about oral hygiene are neglected. In addition, recent studies have shown that oral health care has been shown to be effective in controlling patients' pathogens and systematized care protocols have optimized service delivery. The area, as a whole, of Hospital Dentistry, has the ability to reduce the length of hospital stay of patients, risks of various infections and, without a doubt, provide the quality of life of the sick person. The study aimed to search the literature for fundaments and experiences that would support the discussion about the importance of oral health care of patients hospitalized. The databases used for article tracking were MEDLINE, BBO Odontologia / LILACS and Scielo, using the combination of the terms "oral health"; "inpatients" *; "intensive care units" and "hospital dentistry". Oral pathologies are important sources of systemic of the patient worsening and must be prevented and resolved by a dental surgeon trained to work in a hospital environment. It is worth of note that the conduct protocols are efficient to accelerate and give health care of quality to hospitalized patients.
\end{abstract}

Keywords: Oral Health. Inpatients. Intensive Care Units. Hospital Dentistry.*Admitted Patients. 


\section{INTRODUÇÃO}

A assistência à saúde no Brasil enfrenta inúmeros entraves para sua correta existência, sejam esses entraves de cunho financeiro, político, ou ético. Logo, faz-se cada vez mais pertinente o debate acerca do atendimento holístico ao paciente. Nesse contexo, o holismo defendido pela humanização da saúde requer assistir o paciente em todos os seus aspectos, sejam eles patológicos ou físicos. Portanto, a higiene bucal desses pacientes deve ser avaliada junto com os padrões corporais de saúde, uma vez que, muitas infecções sistêmicas podem ser iniciadas pela cavidade oral (LIMA et al., 2011).

Nessa ótica, o indivíduo hospitalizado, além de necessitar da assistência médica, também necessita da assistência odontológica, para avaliar patologias orais que podem contribuir para o rebaixamento hemodinâmico do paciente e o deterioramento de seu quadro clínico. Portanto, há necessidade de desfazer a imagem da odontologia hospitalar apenas do tratamento curativo-reabilitador realizado e expandir tal conceito para as atividades educativo-preventivas em unidades hospitalocêntricas (CRUZ; MORAES; TREVISANE, 2014).

Em tal panorama, a assistência holística ao pacietne requer uma equipe multidiscplinar que atenda as necessidades dos indivíduos, logo, o odontólogo deve trabalhar integrado a outros profissionais, como a equipe de enfermagem, médicos e fisioterapeutas. Nessa premissa, em fevereiro de 2008 foi apresentado à Câmara dos Deputados o Projeto de Lei $\mathrm{n}^{\circ}$ 2.776/2008, que estabelece como obrigatória a presença do cirurgião-dentista nas equipes multiprofissionais das unidades de terapia intensiva (UTI), para cuidar da saúde bucal dos pacientes (CFO, 2012). Além disso, determina que os internados em outras unidades hospitalares e clínicas também devem receber assistência desse profissional de saúde (LIMA et al., 2011).

Existe uma relação importante entre o estado de saúde bucal e aquele da saúde geral, a literatura já estabelece relação entre a presença de determinadas doenças bucais com o aparecimento e/ou a piora de condições sistêmicas. A relação entre doenças bucais e doenças sistêmicas é um ponto muito discutido na literatura. A presença de doenças bucais, como por exemplo, a candidíase, pode ser um agravante da condição sistêmica do paciente. Sendo assim, a presença do CD fornecendo assistência ao grupo médico pode influenciar e ajudar no diagnóstico e tratamento precoce (SIQUEIRA; BATISTA; FERREIRA; AGOSTINI; TORRES, 2014). 
Logo, enfermos hospitalizados necessitam de muito mais atenção profissional, e a saúde oral deve ser ofertada pelo profissional de odontologia. Portanto, ao serem observados os estudos acerca do tema, o artigo objetivou buscar na literatura aqueles que fundamentassem a discussão sobre a importância do cuidado acerca da saúde bucal em pacientes hospitalizados. Ademais, espera-se contribuir de forma ampla com o enriquecimento da literatura acerca do tema, e também com os demais profissionais de saúde sobre a necessidade da atuação do odontólogo na prestação da assistência holística ao paciente hospitalizado.

\section{METODOLOGIA}

O presente estudo tratou-se de revisão bibliográfica, cujo enfoque foi descritivo exploratório, uma vez que esse processo permitiu a análise e a discussão dos artigos sobre a importância da saúde bucal na terapia de pacientes institucionalizados em unidades de saúde.

Tal tema foi escolhido devido ao embasamento fornecido pelo projeto de Lei $\mathrm{n}^{\circ}$ 2.776/2008, que afirma a necessidade da presença do cirurgião-dentista na equipe multidisciplinar de terapia intensa.

Diante disso, a revisão da literatura foi realizada nos bancos de dados Scielo, Medline e BBO - Odontologia / LILACS. Ao acessar tais bancos de dados, foram utilizados descritores para buscar os artigos que compuseram o corpo do estudo, os seguintes descritores foram: "Unidade hospitalar de odontologia"; "Saúde bucal"; "Pacientes Internados"; "Unidades de terapia intensiva" e Odontologia Hospitalar". Após a inserção dos descritores já citados, foram encontrados 27 artigos segundo os critérios de inclusão. Além disso, foram utilizados critérios para selecionar os artigos que iriam compor o estudo. Sendo que 17 artigos foram excluídos e 10 foram utilizados.

O estudo tratou-se de um levantamento bibliográfico, logo, não foi necessária a aprovação do Comitê de ética e Pesquisa - CEP baseada na Resolução $\mathrm{N}^{\circ} 466$ de dezembro de 2012, que trata da pesquisa envolvendo seres humanos, e nem a revisão ética proporcionada pela Comissão de Ética no Uso de Animais - CEUA, que normaliza as pesquisas feitas com animais, portanto, ausenta-se dessas diretrizes. 


\section{REFERENCIAL TEÓRICO}

\subsection{Odontologia no contexto unidades de terapia intensiva}

A Odontologia Hospitalar é uma especialidade odontológica cujo objetivo é a realização de cuidados e procedimentos no âmbito da saúde no contexto hospitalocêntrico. No que tange à assistência em odontologia em serviços instituicionalizados, há registros que datam de 1901, em que cuidados nessa área foram realizados no Hospital Geral da Filadélfia. Os atendimentos ocorrem com a finalidade de promover saúde entre os enfermos e treinar profissionais para que esses desempenhassem a prática do cuidado em saúde bucal, para com os pacientes institucionalizados, afim de evitar doenças (LIMA et al., 2011).

Estudos acerca do tema evidenciaram que indivíduos instituconalizados possuem tendência a apresentar má higiene bucal, fato que repercute no aumento e na complexidade do bofilme dental, aliado, muitas vezes à proliferação de fungos, como a Candida albicans. Nesse panorama, a falta de higiene bucal acarreta o surgimento de diversas patologias orais, como: xerostomia, periodontite e gengivite, que são capazes de potencializar focos de infecções e que propiciarem maiores riscos de complicações locais e sistêmicas (CRUZ; MORAES; TREVISANE, 2014).

Paralelo a isso, compete ao cirurgiã-dentista especializado em Odontologia Hospitalar, internar e assistir pacientes em hospitais públicos e privados, com ou sem caráter filantrópico, respeitando as normas técnico-administrativas das instituições. Estudos afirmam que o profissional especializado em Odontologia Hospitalar pode trabalhar como consultor da saúde bucal e/ou prestador de serviços, seja em nível ambulatorial ou regime de internação, sempre com o objetivo de colaborar, oferecer e agregar forças ao que caracteriza a nova identidade hospitalar (CFO, 2012).

\subsection{Surgimento de doenças orais e sua relação com a gênese de patologias sistêmicas}

O organismo humano é um conjunto que funciona como um todo, logo, desde épocas passadas era imaginável e associável que patologias orais pudessem desencadear complicações sistêmicas, as primeiras suspeitas de tal relação entre doenças bucais e sistêmicas nos registros datam de 110 a.C. Desde então, muitos pesquisadores se empenham em obter resultados que comprovem essa relação, afim de evidenciar que doenças como 
periodontite e outras atuam como disseminação de patógenos com efeito sistêmico (LIMA et al., 2011).

A cavidade oral é um grande meio de comunicação entre o meio externo e o organismo, logo, é colonizada por vários microrganismos que, em contato com pacientes imunocomprometidos, podem descompensar hemodinamicamente a saúde do paciente, piorando a qualidade de vida e retardando seu tempo de internação. Portanto, a manutenção da saúde bucal dos pacientes hospitalizados é importante para evitar a proliferação de bactérias que, além de comprometer o bem estar dos mesmos, é capaz de danificar órgãos vitais agravando o quadro clínico e, consequentemente, estendendo a sua estadia na UTI.

Em pacientes internados, o cuidado odontológico e as práticas de promoção de saúde ajudam na prevenção e/ou no restabelecimento do quadro sistêmico do paciente, contribuindo para a diminuição de infecções respiratórias, diminuição do uso de medicamentos como antibióticos e consequente taxa de mortalidade. Tais medidas irão causar uma diminuição dos gastos com internação. Na maior parte das vezes, pacientes na UTI apresentam higiene oral precária em função de diversos fatores adicionais relacionados como:

a diminuição da limpeza natural da boca promovida pela mastigação, a movimentação da língua e das bochechas, além da redução do fluxo salivar pelo uso de alguns medicamentos. Em vários casos, há também a existência do tubo traqueal, que prejudica o acesso à cavidade bucal, aumentando a presença do biofilme. Com o tempo de internação se estendendo, haverá o favorecimento da colonização bucal de patógenos respiratórios mais resistentes aos antimicrobianos. A existência da placa bacteriana na cavidade oral pode influenciar as condutas médicas, devido aos fatores de virulência dos microrganismos que nela se deparam, os quais podem ser acentuados pela presença de outras alterações bucais como a doença periodontal, cáries, necrose pulpar, lesões em mucosas, dentes fraturados ou infectados, traumas provocados por próteses fixas ou móveis que podem acarretar para o paciente, implicações na sua condição sistêmica. A complexidade do biofilme bucal e da doença periodontal associada, que corre o risco de se agravar com o tempo de internação, pode ser uma fonte de pneumonia nosocomial. Ela requer atenção especial, pois é a segunda causa de infecção hospitalar e causadora de taxas significativas de morbidade e mortalidade em pacientes de todas as idades. Atinge de $10 \%$ a $15 \%$ das infecções hospitalares, sendo que de $20 \%$ a $50 \%$ dos pacientes afetados por esse tipo de pneumonia vão a óbito (SILVA; AMARAL; CRUZ; SALES, 2017) 


\subsection{A importância do odontólogo na unidade de terapia intensiva}

Os pacientes admitidos nas unidades de terapia intensiva (UTI) frequentemente não contam com assistência à saúde bucal, o que provoca um incremento direto nos problemas de saúde bucal relacionados com morbidade e mortalidade mais elevadas. Uma má saúde bucal pode levar a problemas clínicos, como a disseminação local de infecções, infecções do trato respiratório, maiores custos da admissão à UTI, maior utilização de medicamentos como antibióticos, o que favorece o estabelecimento de resistência bacteriana e infecções oportunistas. A presença de um profissional da odontologia ajuda a manter a adesão aos protocolos de saúde bucal, além de apoiar e dar assistência à equipe para enfrentar as eventuais dificuldades durante os cuidados ao paciente. É também importante salientar a associação entre treinamento adequado da equipe e a presença de um profissional em odontologia na rotina da UTI. Tais resultados são consonantes com outros estudos. Portanto, sugere-se que a presença de um dentista na rotina da unidade de terapia intensiva e a implantação de protocolos institucionais com adequado treinamento da equipe podem influenciar positivamente em sua atitude e levar a uma prática mais coerente de cuidados bucais na unidade de terapia intensiva. ( FRANCISCO et al., 2017)

Em análise do expoto, é visível a necessidade de um profissional de odontologia no serviço de UTI para sanar possíveis causas de complicações sistêmicas, para que possa ser feito o diagnóstico das alterações bucais e consequente auxílio da terapêutica médica; sejam em procedimentos de emergência como traumas ou abcessos, procedimentos que previnam o agravamento da doença sistêmica ou estabelecimento de uma infecção hospitalar e procedimentos curativos, como a adequação do meio bucal do paciente crítico. Ademais, o dentista deve preparar a equipe de Odontologia Hospitalar, incluindo nos procedimentos equipamentos, materiais e instrumentais adequados ao atendimento e a um preparo profissional especializado (LIMA et al., 2011).

\subsection{Como administrar o cuidado para com a saúde bucal de pacientes hospitalizados}

O cuidado com o paciente hospitalizado depende da interação do trabalho multiprofissional, resultado da soma de pequenos cuidados parciais que se complementam. Em se tratando do ambiente hospitalar, a promoção de saúde bucal visa à assistência humanizada e integral ao paciente durante a internação, proporcionando conhecimento e motivando-o e a seus acompanhantes na geração de bons hábitos. Essas ações têm se 
mostrado importantes na incorporação do hábito de higiene bucal dos pacientes à rotina hospitalar, reduzindo o biofilme dentário e, consequentemente, o risco de infecções provenientes da microbiota bucal. Além disso, já se sabe que grande parte das doenças sistêmicas apresenta manifestações bucais que predispõem ao desenvolvimento de processos patológicos, tornando o equilíbrio saúde-doença muito mais frágil. (ROCHA, AMANDA; FERREIRA, EFIGÊNIA et al; 2014)

Junto a isso, estudos evidenciaram que ao desenvolver protocolos que objetivam o tratamento e o controle das doenças bucais de forma rápida e adequada ocorre, consequentemente, a redução da morbimortalidade e a melhora na qualidade de vida do paciente, bem como a diminuição no tempo de hospitalização. Dentre esses, o mais difundido é o Protocolo de Treinamento da Enfermagem para Higiene Oral, que orienta o profissional de enfermagem a respeito da higienização mecânica através da escovação bucal, com a escova a $45^{\circ}$ em direção ao colo dentário e o sulco gengival com ligeiras vibrações nos dentes posteriores e, além de higienização da mucosa com gaze úmida e escovação de língua (BARBOSA; RIBEIRO; CALDO-TEIXEIRA, 2011).

Ressalta-se que, a instituição de medidas de higiene bucal é importante para os pacientes que se encontram hospitalizados. Para aqueles que conseguem fazer a própria higiene, orientações devem ser passadas pelos profissionais que o atendem, especialmente a equipe de enfermagem, que deve ser treinada e capacitada por um cirurgião-dentista para execução de tal tarefa. (SOUSA et al; 2017)

\section{RESULTADOS E DISCUSSÃO}

Tabela 1 - Principais resultados obtidos através dos artigos selecionados para revisão

\begin{tabular}{|l|l|l|l|}
\hline Artigo & Título & $\begin{array}{l}\text { Autores, país e } \\
\text { ano de publicação }\end{array}$ & Principais resultados \\
\hline
\end{tabular}




\begin{tabular}{|c|c|c|c|}
\hline 1 & $\begin{array}{l}\text { A importância da saúde } \\
\text { bucal na ótica de } \\
\text { pacientes } \\
\text { hospitalizados }\end{array}$ & $\begin{array}{l}\text { LIMA, Daniela } \\
\text { Coelho de et al, } \\
\text { Brasil, } 2011\end{array}$ & $\begin{array}{l}\text { A pesquisa foi feita com } \\
\text { pacientes hospitalizados. Mais } \\
\text { da metade dos pacientes } \\
\text { internados eram vítimas de } \\
\text { acidente de moto. A maioria dos } \\
\text { entrevistados estava internada } \\
\text { há } 15 \text { dias e afirmou realizar a } \\
\text { higiene bucal somente duas } \\
\text { vezes ao dia. Todos os pacientes } \\
\text { relataram incômodo com a } \\
\text { presença de halitose e } \\
\text { xerostomia. Eles consideraram } \\
\text { importante a presença do } \\
\text { cirurgião-dentista em uma } \\
\text { unidade hospitalar e os mesmos } \\
\text { enfatizaram a importância da } \\
\text { equipe multidisciplinar para } \\
\text { melhorar o atendimento e a } \\
\text { qualidade de vida dos pacientes. }\end{array}$ \\
\hline 2 & $\begin{array}{l}\text { Avaliação clínica da } \\
\text { cavidade bucal de } \\
\text { pacientes internados } \\
\text { em unidade de terapia } \\
\text { intensiva de um } \\
\text { hospital de emergência }\end{array}$ & $\begin{array}{l}\text { CRUZ, Maristela } \\
\text { Kapitski da; } \\
\text { MORAIS, Teresa } \\
\text { Márcia } \\
\text { Nascimento de; } \\
\text { TREVISANI, } \\
\text { Deny Munari, } \\
\text { Brasil, 2014 }\end{array}$ & $\begin{array}{l}\text { A prevalência de infecção } \\
\text { hospitalar foi de } 22 \% \text {. Todos os } \\
\text { pacientes apresentavam } \\
\text { biofilme bucal. O índice de } \\
\text { placa e a saburra lingual } \\
\text { aumentaram de acordo com o } \\
\text { tempo de internação na UTI. }\end{array}$ \\
\hline
\end{tabular}




\begin{tabular}{|c|c|c|c|}
\hline 3 & $\begin{array}{ll}\text { Candidíase } & \text { oral em } \\
\text { pacientes } & \text { internados } \\
\text { em UTI } & \end{array}$ & $\begin{array}{l}\text { Siqueira JSS, } \\
\text { Batista AS, Silva } \\
\mathrm{Jr} \text { A, Ferreira } \\
\text { MF, Agostini M, } \\
\text { Torres SR, Brasil, } \\
2014\end{array}$ & $\begin{array}{l}\text { A candidíase oral é uma } \\
\text { infecção frequente em pacientes } \\
\text { de UTI. Em pacientes graves } \\
\text { pode complicar com a } \\
\text { disseminação da infecção e a } \\
\text { candidemia, estando associada } \\
\text { ao aumento no período de } \\
\text { internação e morte. A presença } \\
\text { do dentista na UTI pode } \\
\text { proporcionar um diagnóstico e o } \\
\text { tratamento precoce dos quadros } \\
\text { de candidíase oral, reduzindo o } \\
\text { risco de infecção sistêmica, } \\
\text { tempo de internação e custos } \\
\text { hospitalares. }\end{array}$ \\
\hline 4 & $\begin{array}{l}\text { A importância do } \\
\text { cirurgião-dentista em } \\
\text { ambiente hospitalar / } \\
\text { The importance of the } \\
\text { surgeon-dentist at } \\
\text { hospital environment }\end{array}$ & $\begin{array}{l}\text { Silva, Isabelle } \\
\text { Oliveira; Amaral, } \\
\text { Fabrício } \\
\text { Rezende; Cruz, } \\
\text { Priscila Miranda } \\
\text { da; Sales, Talita } \\
\text { Oliveira, Brasil, } \\
2017\end{array}$ & $\begin{array}{l}\text { É fundamental a integração do } \\
\text { cirurgião-dentista habilitado em } \\
\text { Odontologia hospitalar dentro } \\
\text { das UTIs, para realização de } \\
\text { medidas preventivas bucais e } \\
\text { para melhoria do quadro clínico } \\
\text { dos pacientes internados. Além } \\
\text { disso, é importante também que } \\
\text { esse profissional atue na } \\
\text { avaliação dos pacientes antes, } \\
\text { no decurso de sua internação e } \\
\text { após seu tratamento sistemico, } \\
\text { já que existe uma correlação } \\
\text { entre as condições de saúde } \\
\text { sistêmica e oral. }\end{array}$ \\
\hline
\end{tabular}




\begin{tabular}{|c|c|c|c|}
\hline 5 & $\begin{array}{l}\text { Influência da presença } \\
\text { de profissionais em } \\
\text { odontologia } \\
\text { protocolos } \\
\text { assistência à } \\
\text { bucal na equíde } \\
\text { enfermagem de } \\
\text { unidade de terapia } \\
\text { intensiva }\end{array}$ & $\begin{array}{l}\text { BLUM, Davi } \\
\text { Francisco Casa et } \\
\text { al., Brasil, } 2017\end{array}$ & $\begin{array}{l}\text { Sugere que, a presença de um } \\
\text { dentista na rotina da unidade de } \\
\text { terapia intensiva e a } \\
\text { implantação de protocolos } \\
\text { institucionais com adequado } \\
\text { treinamento da equipe, podem } \\
\text { influenciar positivamente em } \\
\text { sua atitude e levar a uma prática } \\
\text { mais coerente de cuidados } \\
\text { bucais na unidade de terapia } \\
\text { intensiva. }\end{array}$ \\
\hline 6 & $\begin{array}{l}\text { Odontologia hospitalar: } \\
\text { a atuação do cirurgião } \\
\text { dentista em equipe } \\
\text { multiprofissional na } \\
\text { atenção terciária }\end{array}$ & $\begin{array}{l}\text { ROCHA, } \\
\text { Amanda } \\
\text { Leal e FERREI } \\
\text { RA, Efigênia } \\
\text { Ferreira e, Brasil, } \\
2014\end{array}$ & $\begin{array}{l}\text { A procura pelo profissional } \\
\text { cresceu sensivelmente, o que } \\
\text { aponta para o reconhecimento } \\
\text { da necessidade da atuação do } \\
\text { cirurgião-dentista por parte dos } \\
\text { demais profissionais. Problemas } \\
\text { como a presença de cárie } \\
\text { dentaria, doença periodontal, } \\
\text { mobilidade dentaria, infecções } \\
\text { bucais e outros, começaram a } \\
\text { despertar nos profissionais do } \\
\text { hospital a necessidade de uma } \\
\text { avaliação especifica. }\end{array}$ \\
\hline 7 & $\begin{array}{lr}\text { Conhecimentos } & \mathrm{e} \\
\text { práticas em } & \text { saúde } \\
\text { bucal com crianças } \\
\text { hospitalizadas } \\
\text { câncer }\end{array}$ & $\begin{array}{l}\text { BARBOSA, } \\
\text { Aline May; } \\
\text { RIBEIRO, } \\
\text { Dayane } \\
\text { Machado; } \\
\text { CALDO- } \\
\text { TEIXEIRA, } \\
\text { Angela Scarparo, } \\
\text { Brasil, 2015 }\end{array}$ & $\begin{array}{l}\text { Todos os participantes } \\
\text { consideram importante haver } \\
\text { um CD no setor de oncologia. } \\
\text { Pôde-se concluir que não existe } \\
\text { um protocolo de cuidados com a } \\
\text { higiene bucal de crianças } \\
\text { hospitalizadas com câncer, e } \\
\text { que as manifestações bucais } \\
\text { mais frequentes entre os } \\
\text { pacientes em tratamento } \\
\text { antineoplásico foram: mucosite, } \\
\text { enjoos, vômitos, xerostomia e } \\
\text { ausência de paladar. }\end{array}$ \\
\hline
\end{tabular}




\begin{tabular}{|c|c|c|c|}
\hline 8 & $\begin{array}{lr}\text { A importância } & \text { da saúde } \\
\text { bucal em } & \text { pacientes } \\
\text { hospitalizados: } & \text { uma } \\
\text { revisão } & \end{array}$ & \begin{tabular}{|l} 
RODRIGUES, \\
Anna Luiza Souza; \\
MALACHIAS, \\
Raphael Corrêa; \\
PACHECO, Cinthia \\
Mara da Fonseca, \\
Brasil, 2018
\end{tabular} & $\begin{array}{l}\text { É preciso proporcionar às pessoas } \\
\text { um tratamento integral, sem } \\
\text { separar a boca do restante do } \\
\text { corpo, visto que as afecções bucais } \\
\text { são importantes fontes de } \\
\text { agravamento de doenças } \\
\text { sistêmicas. }\end{array}$ \\
\hline 9 & $\begin{array}{lr}\text { A participação } & \text { do } \\
\text { cirurgião-dentista } & \text { em } \\
\text { equipe de saúde } \\
\text { multidisciplinar na } \\
\text { atenção à saúde da criança } \\
\text { no contexto hospitalar }\end{array}$ & $\begin{array}{l}\text { MATTEVI, Gianina } \\
\text { Salton et al, Brasil, } \\
2011\end{array}$ & $\begin{array}{l}\text { Os resultados evidenciam a ampla } \\
\text { aceitação e a importância da } \\
\text { participação do cirurgião-dentista } \\
\text { no contexto da Unidade como: } \\
\text { membro da equipe para } \\
\text { concretização do conceito de saúde } \\
\text { integral; profissional de apoio à } \\
\text { equipe nos cuidados e na } \\
\text { dinamização e otimização do } \\
\text { trabalho interdisciplinar. Os } \\
\text { participantes percebem como } \\
\text { muito importante e até } \\
\text { fundamental a participação efetiva } \\
\text { do cirurgião-dentista na atenção à } \\
\text { saúde da criança hospitalizada. }\end{array}$ \\
\hline 10 & $\begin{array}{l}\text { A atuação do residente em } \\
\text { Odontologia Hospitalar } \\
\text { neonatal na abordagem } \\
\text { multidisciplinar do SUS: } \\
\text { relato de experiência / } \\
\text { The multidisciplinary } \\
\text { work of the resident in } \\
\text { neonatal hospital dentistry } \\
\text { in the Brazilian } \\
\text { Healthcare System: an } \\
\text { experience report }\end{array}$ & 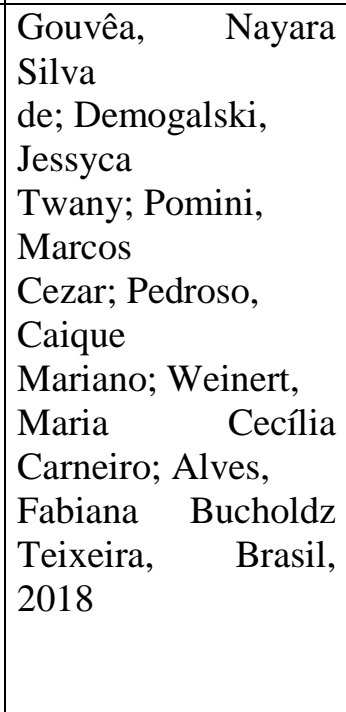 & $\begin{array}{l}\text { Nota-se que existe uma resistência } \\
\text { dos profissionais que possuem } \\
\text { formação voltada ao } \\
\text { ambiente hospitalar no que tange à } \\
\text { inserção do CD } \\
\text { nesse cenário. Nesse sentido, a } \\
\text { residência tem } \\
\text { permitido, além do cuidado } \\
\text { especializado do } \\
\text { sistema estomatognático do RN, } \\
\text { a educação } \\
\text { gradativa da } \\
\text { multiprofissional, por meio da } \\
\text { demonstração prática/teórica da } \\
\text { importância do } \\
\text { exercício odontológico hospitalar. }\end{array}$ \\
\hline
\end{tabular}

No presente artigo, foi observado que a Odontologia em UTI está cada vez mais sendo reconhecida como fundamental à saúde do paciente internado, em contrário à face histórica de que odontologia hospitalar é restrita apenas à área de Cirurgia Bucomaxilofacial. Além disso, as doenças bucais e sistêmicas estão diretamente interligadas; doença periodontal é fator de risco para diabetes e complicações cardíacas (MATTEVI et al., 2011).

Foi unânime entre todos os estudos contemplados que é de sumária importância um profissional dentista dentro dos setores de UTI, uma vez que os pacientes admitidos nesse setor, sejam crianças, recém-nascidos ou adultos, são todos graves e dependentes de cuidado de profissionais de saúde, logo, a manutenção do cuidado bucal deve ser realizada por 
dentistas, confirmando a necessidade do cirurgião- dentista em âmbito hospitalar, já que os pacientes não possuem uma higienização bucal adequada e o controle de placa eficiente.

Em suma, grande parte dos autores mencionados concorda que são necessários mais estudos a respeito da integração do cirurgião - dentista no âmbito hospitalar, e confirmam a relação entre a condição bucal e doenças sistêmicas. Nesse sentido, a inserção do cirurgiãodentista (CD) na equipe hospitalar tem contribuído para a melhora da qualidade de vida do indivíduo e para a redução no tempo de internação e utilização de medicamentos, logo, no custo geral do tratamento. (GOUVÊA, Nayara et al., 2018)

\section{CONSIDERAÇÕES FINAIS}

Foi possível concluir que as patologias bucais são importantes fontes de agravamento sistêmico do paciente, e devem ser prevenidas e solucionadas por um odontólogo especializado em odontologia hospitalar.

Além disso, multidisciplinaridade contida nas UTIs não é completa sem a presença do dentista, já que a grande maioria dos profissionais de saúde que fazem parte do corpo clínico de UTIs não dispõe do conhecimento necessário para o diagnóstico e o tratamento de quais quer condições anormais da cavidade bucal.

Portanto, é de suma importância a presença do cirurgião-dentista no ambiente hospitalar, a fim de melhorar a condição de saúde do paciente e evitar possíveis agravos que, dependendo do caso, podem ser fatais.

\section{REFERÊNCAS}

LIMA, D. C. et al. A importância da saúde bucal na ótica de pacientes hospitalizados. Ciênc. saúde coletiva, Rio de Janeiro, v. 16, supl. 1, p. 1173-1180, 2011.

CRUZ, M. K; MORAIS, T. M. N; TREVISANI, D. M. Avaliação clínica da cavidade bucal de pacientes internados em unidade de terapia intensiva de um hospital de emergência. Rev. bras. ter. intensiva, São Paulo, 26, n. 4, p. 379-383, Dec. 2014.

Conselho Federal de Odontologia. Código de Ética Odontológico. Brasília; 2012. Cap. XI, Artigo 26.

SIQUEIRA J. S. S et al. Agostini M, Torres SR. Candidíase oral em pacientes internados em UTI. Rev Bras Odontol. 2014;71(2):176-9. 
SILVA, I. O et al. . A importância do cirurgião-dentista em ambiente hospitalar / The importance of the surgeon-dentist at hospital environment. Rev. méd. Minas Gerais ; 27: [15], jan.-dez. 2017.

BLUM, D. F. C. et al. Influência da presença de profissionais em odontologia e protocolos para assistência à saúde bucal na equipe de enfermagem da unidade de terapia intensiva. Estudo de levantamento. Rev. bras. ter. intensiva [online]. 2017, vol.29, n.3 [cited 2021-0124], pp.391-393.

ROCHA, A. L.; FERREIRA, E. F. Odontologia hospitalar: a atuação do cirurgião dentista em equipe multiprofissional na atenção terciária. Arq. Odontol. [online]. 2014, vol.50, n.4, pp. $154-160$

BARBOSA, A. M; RIBEIRO, D. M; CALDO-TEIXEIRA, A. S. Conhecimentos e práticas em saúde bucal com crianças hospitalizadas com câncer. Ciênc. saúde coletiva, Rio de Janeiro, v. 15, supl. 1, p. 1113-1122, June 2010.

RODRIGUES, A. L. S. et al. A importância da saúde bucal em pacientes hospitalizados: uma revisão. Revista de Odontologia da Universidade Cidade de São Paulo, [S.1.], v. 29, n. 3, p. 243-248, jul. 2018.

MATTEVI, G. S. et al. A participação do cirurgião-dentista em equipe de saúde multidisciplinar na atenção à saúde da criança no contexto hospitalar. Ciênc. saúde coletiva, Rio de Janeiro, v. 16, n. 10, p. 4229-4236, Oct. 2011.

GOUVÊA, N. S et al. A atuação do residente em Odontologia Hospitalar neonatal na abordagem multidisciplinar do SUS: relato de experiência / The multidisciplinary work of the resident in neonatal hospital dentistry in the Brazilian Healthcare System: an experience report Rev. ABENO_; 18(4): 48-57, 2018.

\section{Como Referenciar este Artigo, conforme ABNT:}

M. O. V. CAVALCANTE, M. S. C. BORBA, M. B. F. SANTOS, A. L. A. GAMA, L. L. LUNAS. A Importância da Manutenção da Saúde Bucal em Pacientes Hospitalizados. Rev. Saúde em Foco, Teresina, v. 8, n. 3, art. 4, p. 54-67, set./dez2021.

\begin{tabular}{|l|c|c|c|c|c|}
\hline \multicolumn{1}{|c|}{ Contribuição dos Autores } & $\begin{array}{l}\text { M. O. V. } \\
\text { Cavalcan } \\
\text { te }\end{array}$ & $\begin{array}{l}\text { M. S. } \\
\text { C. } \\
\text { Borba }\end{array}$ & $\begin{array}{l}\text { M. B. F. } \\
\text { Santos }\end{array}$ & $\begin{array}{l}\text { A. L. A. } \\
\text { Gama }\end{array}$ & $\begin{array}{l}\text { L. L. } \\
\text { Lunas }\end{array}$ \\
\hline 1) concepção e planejamento. & $\mathrm{X}$ & $\mathrm{X}$ & $\mathrm{X}$ \\
\hline 2) análise e interpretação dos dados. & & $\mathrm{X}$ & & $\mathrm{X}$ \\
\hline 3) elaboração do rascunho ou na revisão crítica do conteúdo. & $\mathrm{X}$ & $\mathrm{X}$ & & $\mathrm{X}$ & \\
\hline 4) participação na aprovação da versão final do manuscrito. & $\mathrm{X}$ & $\mathrm{X}$ & & $\mathrm{X}$ & \\
\hline
\end{tabular}

